\title{
Green synthesis and characterization of monodispersed silver nanoparticles using root bark aqueous extract of Annona muricata Linn and their antimicrobial activity
}

\author{
K. M. Ezealisiji ${ }^{1}$ X. S. Noundou ${ }^{2}$ S. E. Ukwueze $^{1}$
}

Received: 18 September 2017/ Accepted: 6 November 2017/Published online: 14 November 2017

(c) The Author(s) 2017. This article is an open access publication

\begin{abstract}
In recent time, various phytosynthetic methods have been employed for the fabrication of silver nanoparticles; these unique metal nanoparticles are used in several applications which include pharmaceuticals and material engineering. The current research reports a rapid and simple synthetic partway for silver nanoparticles (AgNPs) using root bark aqueous extract of Annona muricata and the evaluation of its antimicrobial efficacy against pathogenic microorganisms. The root bark extract was treated with aqueous silver nitrate solution. Silver ions were reduced to silver atoms which on aggregation gave Silver nanoparticles; the biosynthesized AgNPs were characteristically spherical, discreet and stabilized by phytochemical entities and were characterized using ultraviolet visible spectroscopy, transmission electron microscope (TEM) and photon correlation microscopy. The aqueous plant extractAgNPs suspension was subjected to Fourier transform infrared spectroscopy. TEM result for the average particle size is $22 \pm 2 \mathrm{~nm}$. The polydispersity index and zeta-potential were found to be $0.44 \pm 0.02$ and $-27.90 \pm 0.01 \mathrm{mV}, \quad$ respectively (Zeta-Sizer). The antimicrobial evaluation result showed that the synthesized silver nanoparticles at different concentration were very active against the Gram-positive bacteria (B. subtilis, $S$. aureous) and Gram-negative bacteria (K. Pneumonia,
\end{abstract}

K. M. Ezealisiji

Kenneth.ezealisiji@uniport.edu.ng

1 Department of Pharmaceutical Chemistry, Faculty of Pharmaceutical Sciences, University of Port Harcourt, Port Harcourt, Nigeria

2 Department of Chemistry, Rhodes University, Grahamstown, South Africa
E. Coli and Pseudomonas aeruginosa), P. aeruginosa being most susceptible to the anti microbial effect of the silver nanoparticles. Stable silver nanoparticles with antimicrobial activity were obtained through biosynthesis.

Keywords Characterization $\cdot$ Antimicrobial $\cdot$ Silver nanoparticle $\cdot$ Photon correlation microscopy

\section{Introduction}

The emergence of nanotechnology and nanoparticle research has gained ground among interdisciplinary research technologists. Metal nanoparticles have enjoyed great application in healthcare (Siddhartha and Debrata 2009), engineering (Narendra and Uday 2014), and water treatment. The biosynthesis of silver nanoparticles using plant extracts is an interesting area in nanobiotechnology, which is safe, simple, none toxic and eco-friendly with good pharmacological profile. The use in water treatment and purification cannot be overemphasized. In healthcare, silver nanoparticles have enjoyed wide application in areas such as bio-sensing, imaging and drug delivery (Sagar et al. 2011). High mortality rate due to bacterial drug resistance is recently on the increase and the overall result is high cost of medication and prolonged management period; this has recently been countered through the emergence of nanobiotechnology. Fabrication of silver metal nanoparticles employs both physical and chemical method (Saeid et al. 2017), but for interest of this research, the biological method using root bark aqueous extract of Annona muricata was employed in the synthesis of silver nanoparticles. This is a green synthetic method which is eco-friendly alternative to much toxic chemical synthesis (Ram 2014). Phyto-extracts are known to provide mediators for 
biocatalysis such as reducing and capping agents for nanoparticle synthesis. Product yield obtained using phytoextracts is known to be more compared to those of bacterial synthesis, though the particle size range of the latter is known to be finer (Iravanip et al. 2014). Polyphenols are phytochemical compounds with anti-oxidant properties found abundantly in natural plants. There are over 7000 identified polyphenols found in plants (Mamta et al. 2013). Polyphenols play an important role in providing the reducing and capping agents needed in the synthesis of silver metal nanoparticles. In humans, they help in fighting free radicals (Florent et al. 2013). Flavonoids and lignins are example of polyphenols (Oscar et al. 2009). A. muricata has a long history of traditional uses; common names include graviola, guanabana or soursop. Ethnomedicinal uses include anticancer and anti-inflammatory purposes. The plant parts are known to be rich in polyphenols (Bora et al. 2004). To fabricate clinically acceptable and biodegradable nanoparticles, use of injurious chemicals as starting materials should be avoided. Hence, the current research aims at synthesizing silver nanoparticles using aqueous root bark extract of A. muricata, characterization of the synthesized nanoparticles using: UV-visible spectroscopy, transmission electron microscopy (TEM) and photon correlation microscopy (Zeta Sizer) and the evaluation of their antimicrobial activity against representative human pathogenic microorganism.

\section{Experimental section}

\section{Materials}

Silver nitrate, nutrient media, and other reagents used were of analytical grade obtained from Merck, Germany and Oxoid, Hampshire, UK. The medicinal plant, A. muricata employed in the synthesis of the AgNPs was procured from Pharmacognosy garden of the Faculty of Pharmacy, University of Port Harcourt, Nigeria.

\section{Instrumentation}

The morphology and mean particle size of the AgNPs were characterized by transmission electron microscopy (TEM) (VEGAimu GmbH, Germany). Surface resonance plasmon absorption was determined using UV-visible spectrophotometer (Perkin Elmer Lambda 35). The polydispersity index and zeta potential were determined using photon correlation microscope (Mavern Nano ZS, ZS290, and UK). Infrared spectroscopy (FTIR) was performed using the (FTIR) (Shimadzu-8400).

\section{Anonna muricata root extract preparation}

Annona muricata root bark was collected, washed and boiled in double distilled water for $30 \mathrm{~min}$ at $100^{\circ} \mathrm{C}$. The extract was filtered through a cotton cloth sieve to remove insoluble fractions and macromolecules. The filtrate was further re-filtered using $0.45 \mu \mathrm{m}$ sintered glass funnel and the resultant extract was stored in refrigerator at $5{ }^{\circ} \mathrm{C}$ until use. The extract provides basis for the reducing and stabilizing agent.

\section{Synthesis of silver nanoparticles using Anonna muricata root bark aqueous extract}

Silver nitrate in double distilled water afforded the silver ions for the reaction. $100 \mathrm{ml}$ of the root bark aqueous extract was mixed with $150 \mathrm{ml}$ of silver nitrate solution $1.00 \mathrm{mM}$. The reaction mixture was incubated at the temperature of $25^{\circ} \mathrm{C}$ in the dark to avoid photochemical activation of silver nitrate. Silvery-brown colour was observed at the end of $20 \mathrm{~min}$, indicating the formation of AgNPs. The formed product was washed, rinsed and centrifuged with double distilled water dried and stored away from light until used. A. muricata extract was used as control. All experiment was carried out in triplicate.

\section{Characterization of synthesized silver nanoparticles}

The absorption spectra of the silver nanoparticle sample were taken at 190-610 $\mathrm{nm}$ using a UV-visible spectrophotometer (Perkin Elmer Lambda 35) to determine the maximum point of production of silver nanoparticles. Double distilled water was used as blank.

The morphology and size of silver nanoparticles were determined by transmission electron microscopy (TEM). Sample preparation for TEM analysis involves depositing a drop of aqueous silver nanoparticle suspension on a carbon-coated copper grid and allowed to dry at room temperature; the transmission electron micrographs were produced and studied for particle size and morphology.

The average particle size, size distribution by intensity as well as polydispersity index were determined by injecting 1:20 dilution of aqueous silver nanoparticle solution into the U-shaped glass cuvette of the photon correlation microscope. FTIR studies were carried out on the A. muricata extract-silver nanoparticle suspension by employing $\mathrm{KBr}$ pellet technique. The FTIR spectra were generated at a resolution of $4 \mathrm{~cm}^{-1}$ in a transmission mode (4000-400 $\mathrm{cm}^{-1}$ ). 


\section{Antimicrobial activity of synthesized silver nanoparticles}

\section{Microorganisms}

Bacterial and fungal isolates were procured from the Department of Microbiology and Biotechnology of the Faculty of Pharmaceutical Sciences, University of Port Harcourt, Nigeria. The antimicrobial activity experiments were carried out under high ascetic condition.

The method of agar well diffusion assay was employed to investigate the antimicrobial activity of silver nanoparticles (Mukunthan et al. 2011). The tested micro-organisms were seeded in the nutrient agar plates, and then six 4-mm diameter paper discs were saturated with $5,10 \mu \mathrm{g} / \mathrm{ml}$ AgNPs aqueous solution, Chloramphenicol $(0.2 \mathrm{mg} / \mathrm{ml})$, ketoconazole $(0.5 \mathrm{mg} / \mathrm{ml})$, A. muricata aqueous extract and double distilled water (control), respectively. The paper disc was placed on the solidified agar plates and was allowed to incubate at $37{ }^{\circ} \mathrm{C}$ for 24 and $48 \mathrm{~h}$ for bacteria and yeast cultures, respectively. The inhibition zone diameter was measured.

\section{Result and discussion}

The phytosynthesis of metallic silver nanoparticles using plant extracts in comparison with other bio-reducing agents is more yielding in terms of its simplicity (Kumar et al. 2014). The fact remains that the phytochemical principles such as anti-oxidants do not only reduce the $\mathrm{Ag}^{+}$to $\mathrm{Ag}^{0}$ but also are implicated in the capping and stability of the nanoparticles (Vidhu et al. 2011). Hence, root bark of $A$. muricata was exploited for the biosynthesis of novel silver nanoparticles (Fig. 1).

\section{Physichochemical characterization of AgNPs}

\section{Ultraviolet-visible spectroscopy}

One step reaction between silver nitrate solution and root bark aqueous extract of $A$. muricata produced a colour change from yellowish to silvery brown due to the excitation of surface plasmon vibration (Kumari et al. 2016), which clearly indicates the presence of AgNPs. The above reaction afforded silver nanoparticles which were analyzed by UV-visible spectroscopy. The result showed an absorption surface Plasmon peak at the region of $420 \mathrm{~nm}$ after $8 \mathrm{~h}$ (see Fig. 2). The absorption peak was not observed for the silver nitrate solution and aqueous bark extract, respectively.

\section{Result of TEM micrograph of AgNPs}

Total percentage yield of silver nanoparticles obtained after $8 \mathrm{~h}$ was calculated to be $50 \%$. Studies of TEM micrograph of AgNPs gave a general overview of the distribution and the average particle size; the particles were observed to be discreet, monodispersed and topographically spherical (see Fig. 3) with size range between 15.08 and $33.11 \mathrm{~nm}$ with mean particle size $22 \pm 2 \mathrm{~nm}$.

\section{Droplet size, polydispersity index and zeta-potential result}

Photon correlation spectroscopy analysis showed that zeta potential of the silver nanoparticle is $-27.90 \pm 0.10 \mathrm{mV}$ with polydispersity index of $0.44 \pm 0.02$ while the mean particle size was $392.10 \pm 18.56 \mathrm{~nm}$. Some particles were found well below $100 \mathrm{~nm}$ while the majority of the particles were between 150 and $400 \mathrm{~nm}$ though some few particles were recorded at the micrometer range as a result of particle aggregation with time (see Fig. 4a). The nanoparticle size is larger as presented by the DLS; this could only be explained based on the hydrodynamic radius which is not a true size as a result of the hydration layer around the particles as well as the presence of capping and stabilizing agents.

Sample analysis

Average size: $392.10 \pm 18.56$

PDI: $0.44 \pm 0.02$

$\mathrm{ZP}:-27.90 \pm 0.10$

\section{FTIR analysis of aqueous root extract of Anonna muricata-AgNPs}

The account of phytochemical involvement of different functional groups in the synthesis of silver nanoparticles is monitored by Fourier transformed infrared spectroscopy. The FTIR spectra (Fig. 5) were recorded for aqueous extract of $A$. muricata-AgNPs suspension. Chemometric profiling of extracts of $A$. muricata afforded phytochemical principles (Patel and Jayvadan 2016), mainly steroids, alkaloids, antioxidants, polyphenols, carbohydrates, glycosides and flavonoids. The band intensities of the test sample were analysed as shown below. The prominent peak at $2958 \mathrm{~cm}^{-1}$ is attributed to the $-\mathrm{NH}$ stretching vibration of amine while the observed peak at $3393 \mathrm{~cm}^{-1}$ is due to the $-\mathrm{OH}$ stretching vibration of hydroxyl functional groups of flavonoids, polyphenols, and alcohols. The prominent peak at $2958 \mathrm{~cm}^{-1}$ is attributed to the $-\mathrm{NH}$ stretching vibration of amine while the observed peak at 
Fig. 1 Mechanism of phytochemical-assisted silver nanoparticle formation and capping

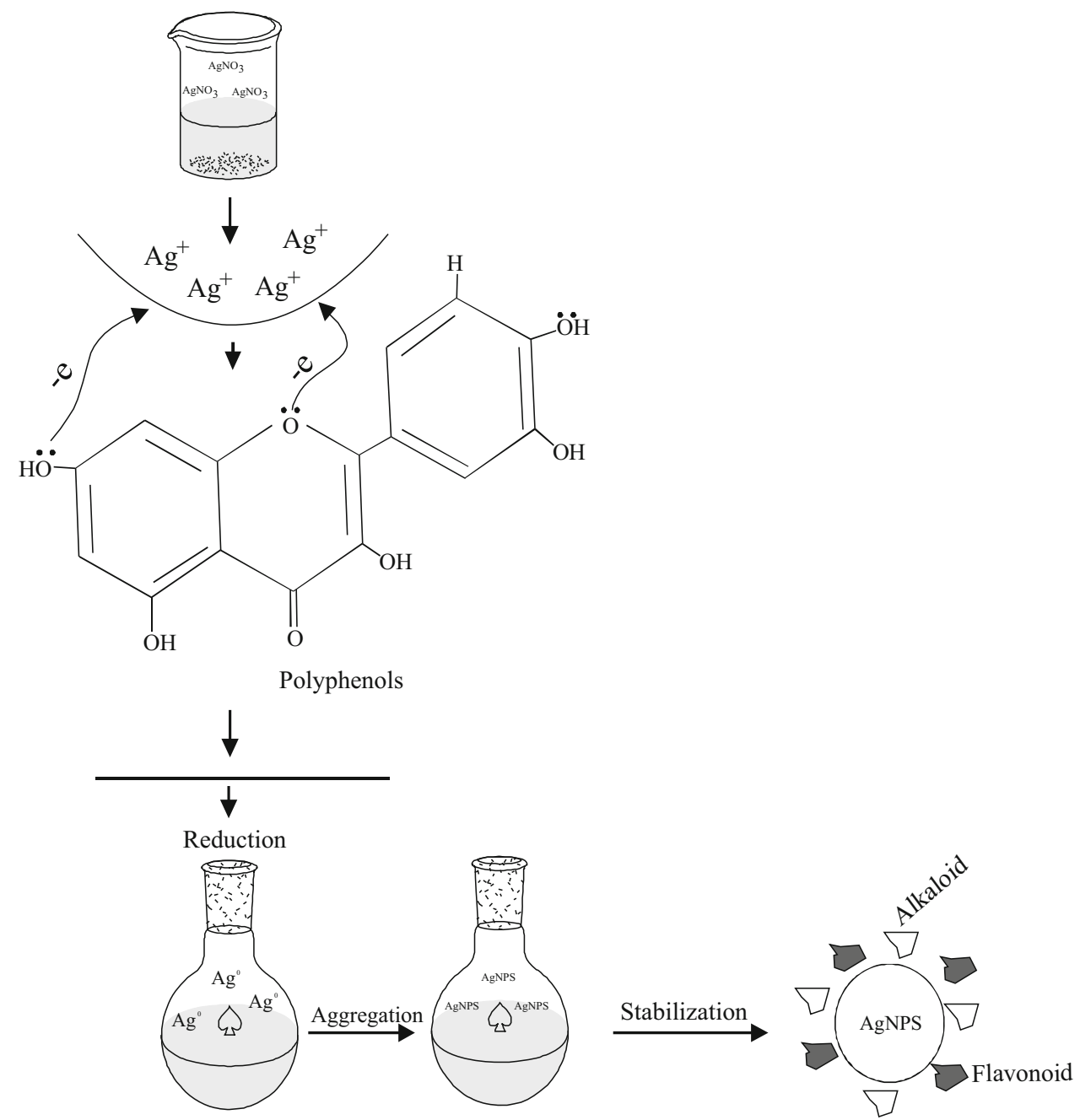

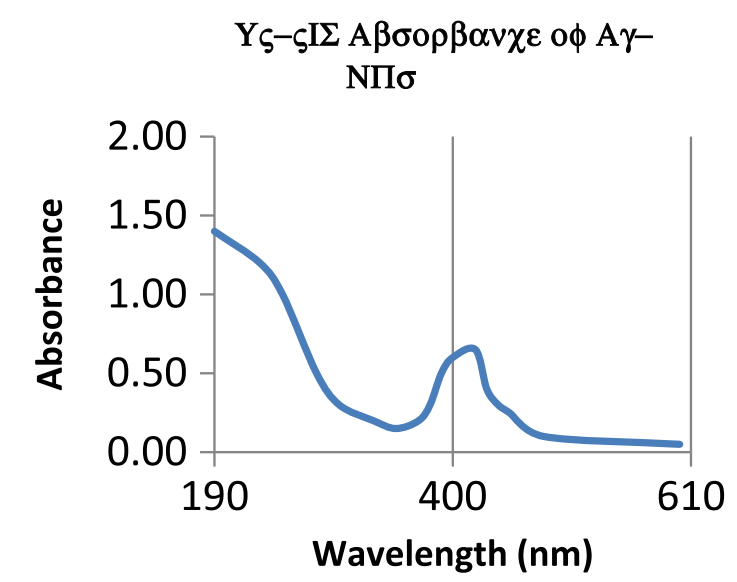

Fig. 2 UV-visible spectroscopic micrograph showing the spectral absorbance of A. muricata stem bark-synthesized AgNPs at $420 \mathrm{~nm}$

$3393 \mathrm{~cm}^{-1}$ is due to the $-\mathrm{OH}$ stretching vibration of hydroxyl functional groups of flavonoids, polyphenols, and alcohols. The peaks at 2919 and $2850 \mathrm{~cm}^{-1}$ are specific to methyl groups or $-\mathrm{CH}$ of aldehydes while the peak at

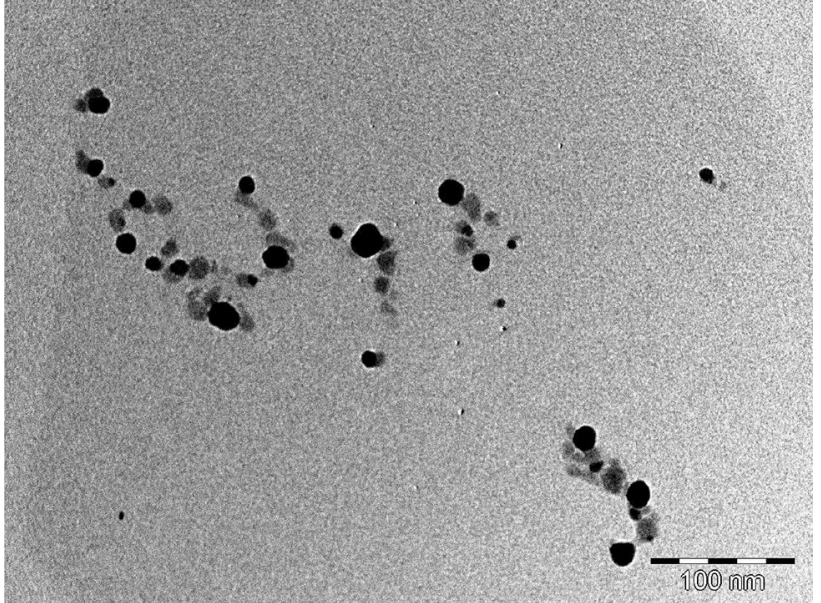

Fig. 3 TEM micrograph revealing the topography, size and monodispersed AgNPs

$1710 \mathrm{~cm}^{-1}$ is attributed to carbonyl stretching vibration of esteric groups. The sharp and strong peak observed at $1659 \mathrm{~cm}^{-1}$ is attributed to the $-\mathrm{OH}$ bending mode or $\mathrm{C}=\mathrm{O}$ 
(a)

Size Distribution by Intensity

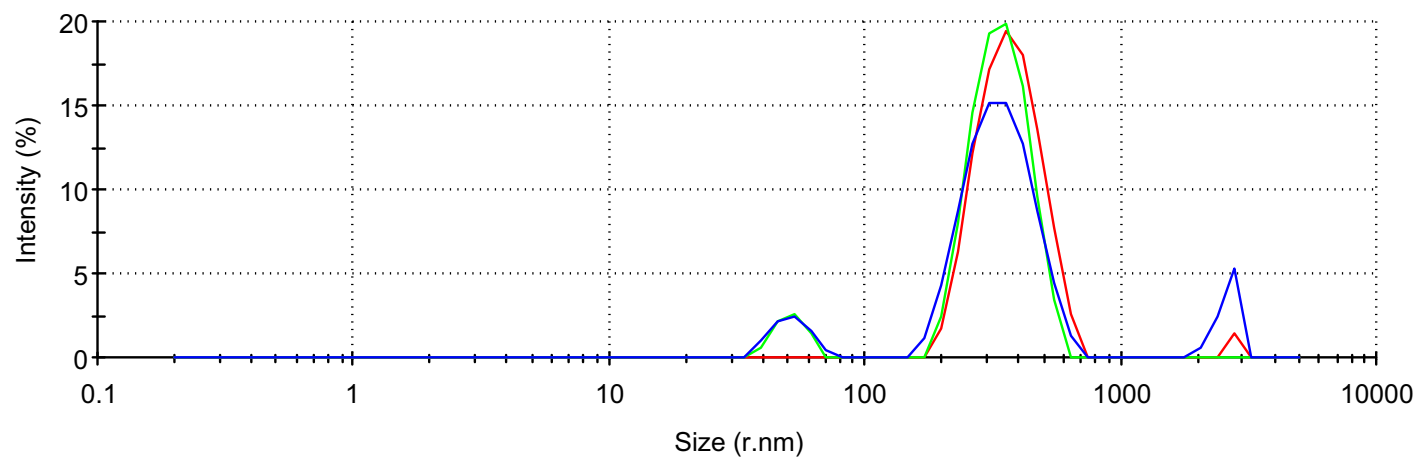

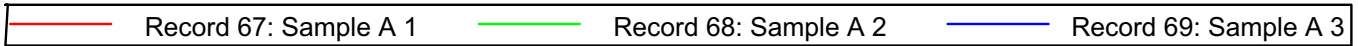

(b)

Size Distribution by Number

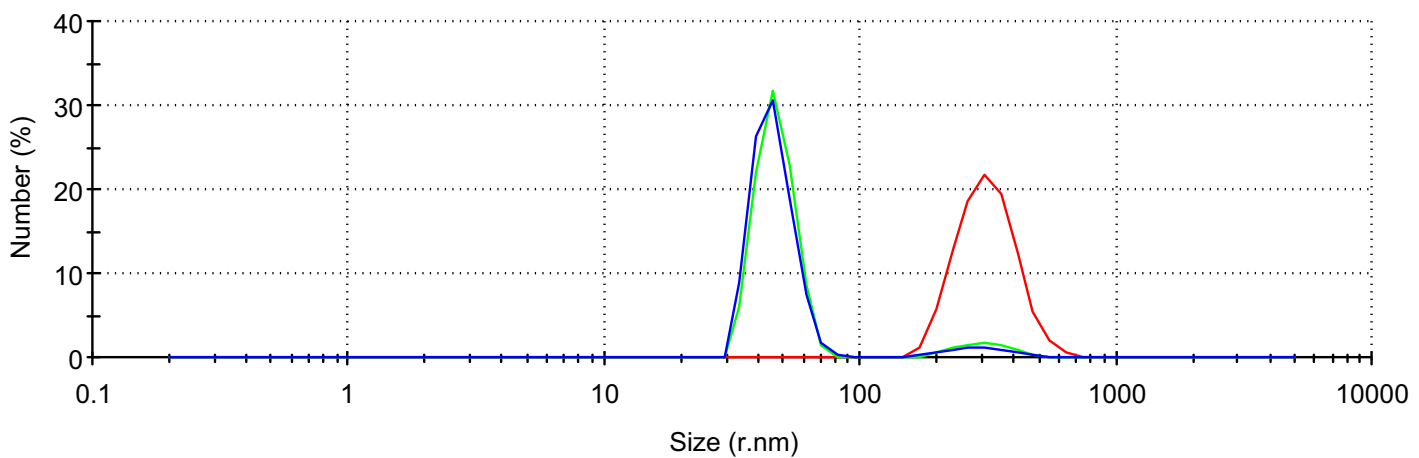

Record 67: Sample A $1 —$ Record 68: Sample A 2

Record 69: Sample A 3

Fig. 4 a Size distribution by intensity for synthesized AgNPs. b Size distribution by number for synthesized AgNPs

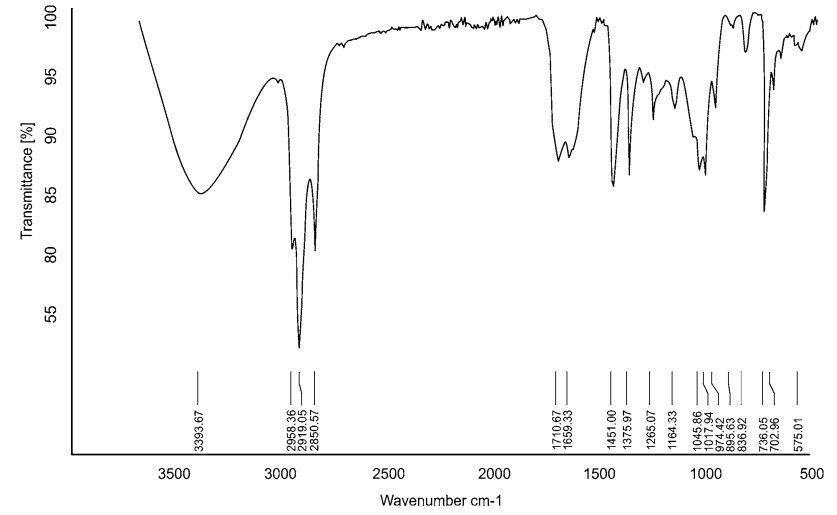

Fig. 5 FTIR spectra showing the functional groups associated with capping and reduction of silver ions to atoms

stretching vibration of carbonyl and carboxylic group of amide I while the slight interaction around $1265 \mathrm{~cm}^{-1}$ is due to amide II vibrations. Antioxidants, alkaloids, flavonoids, tannins, phenols and carbohydrates are the main component of the aqueous extract of the plant root bark
(Ezealisiji and Tamuno 2017). The possible agents that can effectively reduce $\mathrm{Ag}^{+}$to $\mathrm{Ag}^{0}$ and hence aggregates of silver nanoparticles include the antioxidants, especially polyphenols while the alkaloids and flavonoids acted as the stabilizing and capping agents, respectively. Antioxidants are the main component of the aqueous extract of the plant root bark; they are known to possess a signature peak around this region and are implicated in the bioreduction process of the metallic ions $\left(\mathrm{Ag}^{+}\right)$and stabilization of nanoparticles.

\section{Result of AgNPs antibacterial efficacy}

It was observed that both Gram-positive and Gram-negative pathogenic test organisms were highly sensitive to the antimicrobial activity of synthesized AgNPs with a mean zone of inhibition(ZOI) in diameters of 10.00, $15.00 \mathrm{~mm}$ and 12.50, 17.50, $20.00 \mathrm{~mm}$ for B. subtilis, S. aureous, and K. pneumonia, E. coli, and Pseudomonas aeruginosa, respectively, at the dose of $5 \mu \mathrm{g} / \mathrm{ml}$. At higher dose of 


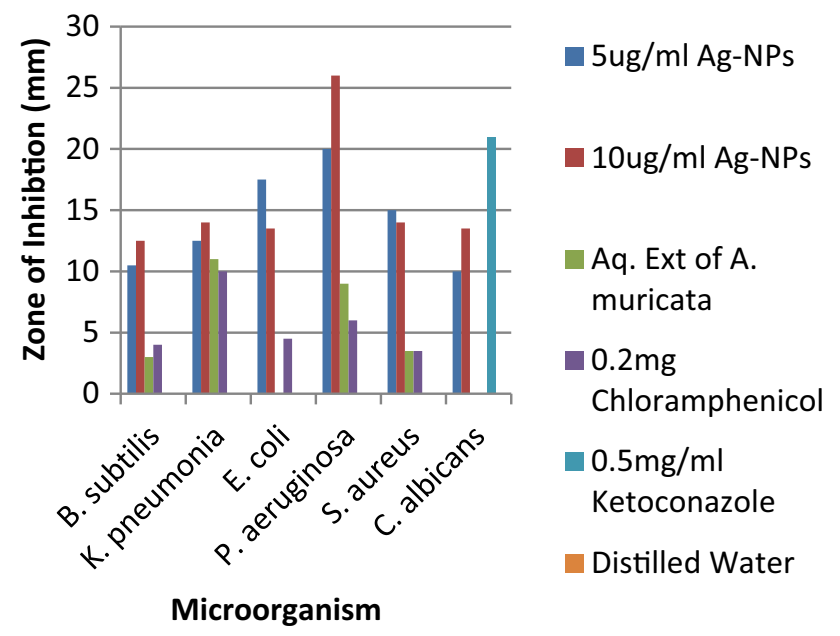

Fig. 6 Inhibition zone diameter for synthesized AgNPS (5, $10 \mu \mathrm{g} /$ $\mathrm{ml}$ ), aqueous A. muricata extract, $0.2 \mathrm{mg} / \mathrm{ml}$ chloroamphenicol, $0.5 \mathrm{mg} / \mathrm{ml}$ ketoconazole and distilled water against pathogenic organisms

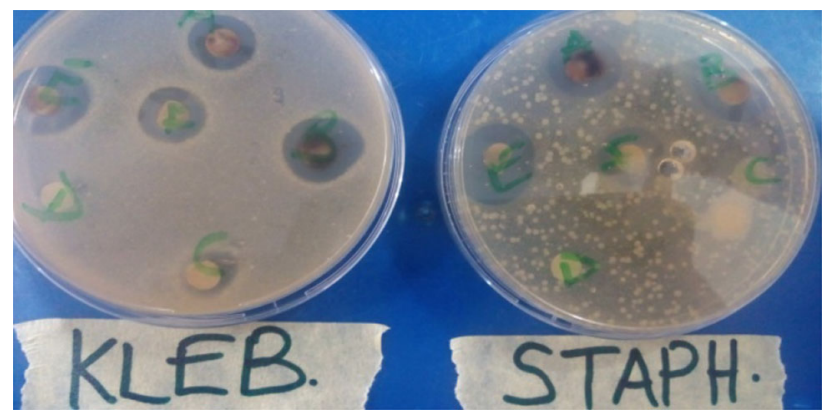

Fig. 7 Determination of Inhibition zone diameter of green synthesized AgNPs 5, $10 \mu \mathrm{g} / \mathrm{ml}$, chloramphenicol, distilled water and Annona muricata root bark extract. Against $K$. pneumonia and $S$. aureus

$10 \mu \mathrm{g} / \mathrm{ml} \mathrm{AgNPs}$, the zone of inhibition was found to be $12.50,14.50 \mathrm{~mm}$ and $14.00,18.50$, and $26.00 \mathrm{~mm}$ for $B$. subtilis, S. aureous and $K$. pneumonia, E. Coli and $P$. aeruginosa, respectively (Fig. 6). From the above result, it was observed that AgNPs inhibit bacterial growth in a dose-dependent manner with $P$. aeruginosa being more susceptible to the antibacterial agent (AgNPs). The synthesized AgNPs have very profound antibacterial efficacy when compared with the standard antibacterial agent used (chloramphenicol). The aqueous root bark extract also showed a remarkable antibacterial activity against some of the tested organisms, $K$. pneumonia and $P$. aeruginosa being more susceptible. At different concentration of AgNPS, the antifungal effect was quite remarkable though less than the effect of ketoconazole (Fig. 6). The silver nanoparticles prepared using A. muricata root bark extract were found to be monodispersed, with better characteristics of nanoparticles; the synthesis rate is faster and the product is less toxic when compared to those of chemical and physical approach. Previous work has shown that chemically synthesized nanoparticles were highly dispersed with lesser particle size range though the antimicrobial assay proved biogenic AgNPS to be more potent antimicrobial agents than chemically synthesized AgNPS (Bawskar et al. 2015). Hence AgNPS synthesized from biogenic A. muricata root bark extract were considered more potent than reviewed products of chemical synthesis. Pharmaceutical research in this area of biosynthesis will prefer a non-toxic product, especially where the product is intended to be used for pharmaceutical, cosmetics, food and medical application (Fig. 7).

\section{Conclusion}

In the reported research work, AgNPs have been synthesized using A. muricata root bark extract. Synthesized AgNPs showed remarkable stability. The UV-visible spectroscopy, TEM and photon correlation spectroscopy analysis confirm the existence of elemental silver and its spherical form. The synthesized AgNPs have been confirmed to show profound activity against pathogenic bacteria. The present research is a simple, rapid, eco-friendly and non-toxic protocol for the synthesis of silver nanoparticles.

Acknowledgements The authors do extend their profound appreciation to management of Department of Chemistry, Rhodes University, Grahamstown, South Africa for granting the research team access to their state of the art Nanotechnology centre.

\section{Compliance with ethical standards}

Conflict of interest The authors report no conflicts of interest.

Open Access This article is distributed under the terms of the Creative Commons Attribution 4.0 International License (http:// creativecommons.org/licenses/by/4.0/), which permits unrestricted use, distribution, and reproduction in any medium, provided you give appropriate credit to the original author(s) and the source, provide a link to the Creative Commons license, and indicate if changes were made.

\section{References}

Bawskar M, Deshmukh S, Bansod S, Gade A, Rai M (2015) Comparative analysis of biosynthesized and chemosynthesized silver nanoparticles with special reference to their antimicrobial activity against pathogens. IET Nanobiotechnol 9(3):107-113

Bora PS, Holschuh HJ, da Silva Vasconeos MA (2004) Characterization of polyphenol oxidase of soursop (Annona muricata L.) fruits and comparative study of its inhibition in enzyme extract and in pulp. Client Technol Aliment 4(4):267-273

Ezealisiji KM, Tamuno EB (2017) Comparative evaluation of the phenolic and antioxidant properties of the leaves, root, stem bark 
and root bark of Annona muricata (Annonaceae). J Pharm Phytochem 6(2):274-278

Florent DM, Vincent L, Jerome C, Robarto L, Jean-Luc D, Younn O, Patric T (2013) Free radical scavenging by natural polyphenols: atom versus electron transfer. J Phys Chem A 117(10):2082-2092

Iravanip S, Korbekandi H, Mirmohammadi SV, Zolfaghri B (2014) Synthesis of silver nanoparticles: chemical, physical and biological method. Res Pharm Sci 9(6):385-406

Kumar R, Smita K, Cumbal L, Debut A (2014) Synthesis of silver nanoparticles using Sacha inchi (Plukenetia volubilis) leaf extracts. Saudi J Biol Sci 21(6):605-609

Kumari J, Mamta B, Ajeet S (2016) Characterization of silver nanoparticles synthesized using Urtica dioica Linn. Leaves and their synergistic effect with antibiotics. J Radiat Res Appl Sci 9(3):217-227

Mamta S, Jyoti S, Rajeev N, Dharmendra S, Abhishek G (2013) Phytochemisry of medicinal plants. J Pharmacogn Phytochem $1(6): 45-52$

Mukunthan KS, Elumalai EK, Trupti N, Ramachandra M (2011) Catharantus roseus: a natural source for the synthesis of silver nanoparticles. Asia Pac J Trop Biomed 1(4):270-274

Narendra K, Uday M (2014) Biosynthesis of metal nanoparticles: a review. J Nanotechnol 4(2):62-70
Oscar MM, Correra YM, Niño J (2009) Anti oxidant activity of plant extract from Colombian flora. Braz $J$ Pharmacogn 19(2A):382-387

Patel S, Jayvadan KP (2016) A review on a miracle fruits of Annona muricata. J Pharmacogn Phytochem 5(1):137-148

Ram P (2014) Synthesis of silver nanoparticles in photosynthetic plants. J Nanopart 4(1):32-40

Saeid A, Fatemeh K, Necip A, Elen RS, Ehian FM, Elahe A (2017) Synthesis of CdO nanoparticles using direct chemical precipitation method. J Biomed Nanotechnol 47(3):120-128

Sagar RM, Amol BD, Sachin P, Chatan MB (2011) Nanoparticles: emerging carriers for drug delivery. Saudi Pharm J 19(3):129-141

Siddhartha S, Debrata D (2009) Applying nanotechnology to human health: revolution in biomedical sciences. J Nanotechnol 2(1):54-59

Vidhu VK, Aromal A, Philip D (2011) Green synthesis of silver nanoparticles using Macrotyloma uniflorum. Acta Part A Mol Biomol Spectrosc 83(9):392-397

\section{Publisher's Note}

Springer Nature remains neutral with regard to jurisdictional claims in published maps and institutional affiliations. 